\title{
A Lower Bound for the Mass of a Random Gaussian Lattice*
}

\section{David Brydges ${ }^{1}$ and Paul Federbush ${ }^{2}$}

1 Rockefeller University, New York, New York 10021, USA

2 Department of Mathematics, University of Michigan, Ann Arbor, Michigan 48109, USA

\begin{abstract}
We give a criterion that the two point function for a Gaussian lattice with random mass decay exponentially. The proof uses a random walk representation which may be of interest in other contexts.
\end{abstract}

Random mass gaussian lattices are lattice systems where the single site distribution has the form

$$
\left(\int_{0}^{\infty} d \sigma(a) e^{-a \phi^{2}}\right) d \phi
$$

An example is $\frac{d \phi}{1+\phi^{2}}$. Related systems have been discussed quite frequently, at least in one dimension [1]. ${ }^{1}$

Let $d \sigma(a)$ be a Borel measure on $(0, \infty)$ such that

$$
\int d \sigma(a)(1+a)^{-1 / 2}<\infty \text {. }
$$

For $\mu \geqq 0$, define

$$
d m_{\mu}(\phi)=\left(\int d \sigma(a) e^{-(a+\mu) \phi^{2}}\right) d \phi .
$$

Let $L_{\infty} \subset \mathbb{R}^{d}$ be a unit lattice centered on the origin, parallel to the coordinate axes. $L$ denotes the finite part of $L_{\infty}$ contained in the box $\prod_{j=1}^{d}\left[-l_{j}+1 / 2, l_{j}-1 / 2\right]$ where $\left(l_{j}\right)$ are given integers. On the space $\mathbb{R}^{|L|}$, where $|L|$ denotes the number of lattice points in $L$, define the probability measure

$$
\begin{aligned}
d P_{L, \mu} & =Z_{L, \mu}^{-1} \prod_{l \in L} d m_{\mu}\left(\phi_{l}\right) e^{\left(\phi, A_{D} \phi\right)}, \\
\left(\phi, \Delta_{D} \phi\right) & =-\sum_{l, l^{\prime}}\left(\phi_{l}-\phi_{l}\right)^{2} .
\end{aligned}
$$

* Supported by N.S.F. Grants PHY 76-17191, MPS 10751

1 The thermodynamic limit is taken after integrating over the masses, in this paper 
$Z_{L, \mu}$ is the normalisation. $A_{D}$ is the finite difference laplacian with Dirichlet boundary conditions, so the sum in (4) is over nearest neighbor lattice points in $L_{\infty}$ and $\phi_{l} \equiv 0$ if $l \notin L$.

The measure for the random mass gaussian lattice is to be obtained by taking limits $L>L^{\infty}, \mu>o$ in that order. By Griffith inequalities the moments of $d P_{L, \mu}$ are monotone increasing as $|L|$ increases and $\mu$ decreases, therefore existence reduces to uniform upper bounds.

In order to state and prove the theorem, we define for $n \geqq o$,

$$
\begin{aligned}
d m_{\mu, n}(\phi) & =\left(\int d \sigma(a)\left(\frac{2 d}{2 d+\mu+a}\right)^{n} e^{-(a+\mu) \phi^{2}}\right) d \phi, \\
Z_{T, \mu, n} & =\int \prod_{l \in T} d m_{\mu, n}\left(\phi_{l}\right) e^{\left(\dot{\phi}, \Delta_{T} \phi\right)} .
\end{aligned}
$$

$T$ is a lattice wrapped around a torus. Given $L$, it is defined by identifying the boundary points of $L_{\infty} \cap \prod_{j=1}^{d}\left[-l_{j}-1 / 2, l_{j}+1 / 2\right]$ in the obvious way. $\Delta_{T}$ is the finite difference laplacian with periodic boundary conditions defined by an equation like (4) in which $l, l^{\prime}$ are summed over nearest neighbors in $T$. Corresponding to $d P_{L, \mu}$ is a measure $d P_{T, \mu}$ obtained by replacing $L$ by $T, \Delta_{D}$ by $\Delta_{T}$ and $Z_{L, \mu}$ by $Z_{T, \mu, 0}$. The periodic pressure is defined by

$$
P_{\mu, n}=\lim _{L>L_{\infty}}|T|^{-1} \log Z_{T, \mu, n} \text {. }
$$

Theorem. The two point function

$$
\lim _{\mu>0} \lim _{L \rightarrow L_{\infty}} \int d P_{L, \mu} \phi_{l} \phi_{l^{\prime}}
$$

exists and is $0\left(e^{-M|l-l|}\right)$ as $\left|l-l^{\prime}\right| \rightarrow \infty$ for some $M>0$ provided

$$
A \equiv \liminf _{\mu \rightarrow 0}\left(P_{\mu, 0}-P_{\mu, 1}\right)>0 . \quad(M \geqq A) .
$$

Remarks. The inequality $A \geqq 0$ is an obvious consequence of the definitions. We think $A>0$ will hold for $d \geqq 3$, provided $d \sigma(a) \neq \delta(a)$. For $d<3$, one must either place additional restrictions on $d \sigma(a)$ near $a=0$ to ensure even existence as $\mu \rightarrow 0$ or look at correlations of different quantities such as $\operatorname{grad} \phi$. The proof will use the following proposition ${ }^{1}$ which may also be of interest.

Proposition. Let $b=\left(b_{l}\right)$ be a strictly positive function on $T$. Then

$$
\left(b-\Delta_{T}\right)_{l, l^{\prime}}^{-1}=\sum_{\omega} \prod_{l \in T}\left(2 d+b_{l}\right)^{-n(l, \omega)}
$$

$\omega$ is summed over all random walks on T of arbitrarily many nearest neighbor steps starting at $l$, ending at $l . n(l, \omega)$ is the number of times $\omega$ hits $l$. The left hand side means the l, $l^{\prime}$ entry of the matrix inverse.

Proof of Proposition. $\left(\Delta_{T} \equiv \Delta\right.$.)

$$
\begin{aligned}
(b-\Delta)^{-1} & =(b+2 d-2 d-\Delta)^{-1}, \\
& =(b+2 d)^{-1}+(b+2 d)^{-1}(2 d+\Delta)(b+2 d)^{-1}+\ldots .
\end{aligned}
$$

1 This is a reformulation of a well known theorem in random walk 
This is the resolvent expansion in the off diagonal elements. The last line can be rewritten as the right hand side of the proposition because $(2 d)^{-1}(2 d+4)$ generates random walk. (It is a matrix with positive elements which sum to one.)

Proof of Theorem by a Griffiths inequality

$$
0 \leqq \int d P_{L, \mu} \phi_{l} \phi_{l^{\prime}} \leqq \int d P_{T, \mu} \phi_{l} \phi_{l^{\prime}} .
$$

Substitute for $d m_{\mu}(\phi)$ using (2). The right hand side becomes $\left(Z \equiv Z_{T, \mu, 0}, \Delta \equiv \Delta_{T}\right)$

$$
\begin{aligned}
& 1 / 2 Z^{-1} \int \prod_{k \in T} d \sigma\left(a_{k}\right) \operatorname{det}^{-1 / 2}(2(\mu+a-\Delta))(\mu+a-\Delta)_{l, l^{\prime}}^{-1}, \\
& =1 / 2 Z^{-1} \int \prod_{k \in T} d \sigma\left(a_{k}\right) d \phi_{k} \exp \left\{-\left(\mu+a_{k}\right) \phi_{k}^{2}\right\}(\mu+a-\Delta)_{l, l^{\prime}}^{-1} e^{(\phi, \Delta \phi)} .
\end{aligned}
$$

Therefore by the proposition

$$
\begin{aligned}
\int d P_{T, \mu} \phi_{l} \phi_{l^{\prime}}= & 1 / 2 \sum_{\omega} \prod_{k \in T}(2 d)^{-n(k, \omega)} \\
& \cdot Z^{-1} \int \prod_{k \in T} d m_{\mu, n(k, \omega)}\left(\phi_{l}\right) e^{(\phi, A \phi) .}
\end{aligned}
$$

We now apply Osterwalder-Schrader positivity in the form of the chess board estimate [2] (Lemma 4.5) to show

$$
\begin{gathered}
\int \prod_{l \in T} d m_{\mu, n(l, \omega)}\left(\phi_{l}\right) e^{(\phi, \Delta \phi)} \\
\leqq \prod_{\ell \in T}\left(Z_{T, u, n l(l, \omega)}\right)^{\frac{1}{|T|}} .
\end{gathered}
$$

Combine (10), (13), and (14) and pass to the limit $L>L_{\infty}$ using definition (7).

$$
\begin{aligned}
\lim _{L \varkappa_{\infty}} \int d P_{L, \mu} \phi_{i} \phi_{l^{\prime}} \leqq & 1 / 2 \sum_{\omega} \prod_{l \in L_{\infty}}(2 d)^{-n(l, \omega)} \\
& \cdot \exp \left(P_{\mu, n(l, \omega)}-P_{\mu, 0}\right) \\
\leqq & \operatorname{const} e^{-A\left|l-l^{\prime}\right|} .
\end{aligned}
$$

The last inequality is using the fact that each $\omega$ must visit at least $|l-l|$ lattice points and $P_{\mu, n} \geqq P_{\mu, 1}$ for $n \geqq 1$. Proof concluded.

Remarks. (1) Representations like (13) can be obtained for $n$ point functions.

(2) By using

$$
\delta\left(\phi^{2}-1\right)=\frac{1}{2 \pi} \int d a e^{i a\left(\phi^{2}-1\right)}
$$

representations like (13) can be obtained for rotators with $n$ components. Despite the complex numbers in (13) one still obtains positive measures $d m_{\mu, n}(\phi)$. 


\section{References}

1. Lieb, E., Mattis, D.: Mathematical physics in one dimension, pp. 119-196. New York: Academic Press 1967

2. Fröhlich,J.: Phase transitions, Goldstone bosons, and topological superselection rules. Acta Phys. Austriaca, Suppl. XV, 133 (1976)

Communicated by A. Jaffe

Received March 29, 1978; in revised form May 8, 1978 